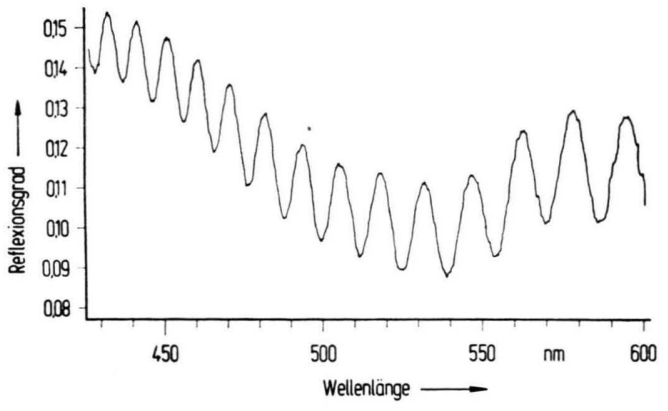

Abb. 1. Reflexionsgrad einer unbelichteten Kodak HR-Platte.

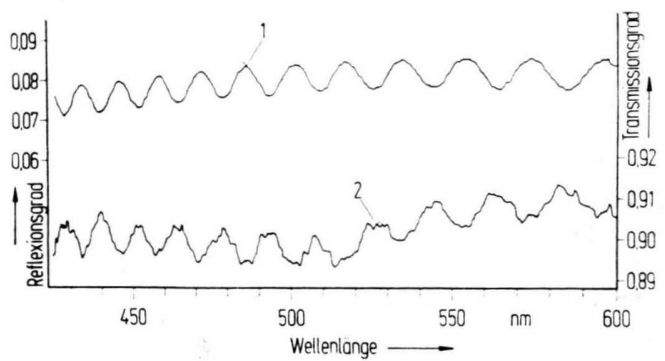

Abb. 2. Reflexionsgrad (Kurve 1) und Transmissionsgrad (Kurve 2) einer unbelichteten, ausfixierten Kodak HR-Platte.

rie der Interferenzen in dünnen Schichten durch die Gleichung

$$
2 n d=k \lambda, \quad k=1,2,3, \ldots
$$

beschreiben, wobei $n$ die Brechzahl der photographischen Schicht, $d$ die Dicke der Schicht und $\lambda$ die Wellenlänge ist. Die Schichtdicke wurde mit einem Düsenmeßbügel für die unbelichtete Schicht zu $6,0 \mu \mathrm{m}$ bestimmt.
Die Brechzahl solcher Schichten ist direkt nur schwer mit hinreichender Genauigkeit zu messen. Die graphische Auswertung nach Gl. (1) ergibt eine Brechzahl von 1,73 für die unbelichtete Schicht der HR-Platte. Unter der Annahme, daß die Feinstruktur auf Interferenzen zurückzuführen ist, kann man nach Formeln von VAšIČEK ${ }^{5}$ aus der Differenz des Reflexionsgrades benachbarter Minima und Maxima eine untere Grenze für die Brechzahl der Schicht errechnen. Sie beträgt $n=1,59$; dieser Wert stimmt innerhalb der Fehlergrenzen (Dickenmessung, Unsicherheit etwa 5\%) mit dem Wert überein, den man nach Gl. (1) erhält.

Aus den Versuchen muß geschlossen werden, daß die von uns beobachtete Feinstruktur durch Interferenzen entsteht.

Weiterhin konnte gezeigt werden, daß mit wachsender Schichtdicke (Kodak MR-Platte, ausfixierte AgfaScientia-Platte 44 A 56) der Abstand der Extremwerte voneinander geringer und die Auflösung der Feinstruktur schwieriger werden. Durch starke Streuung bzw. Absorption in der Schicht (unbelichtete Agfa-ScientiaPlatte 44 A 56) verschwindet die Feinstruktur vollständig. Demnach sind geringe Schichtdicken und geringe Absorption sowohl für die Beobachtung der Interferenzen als auch für die Beobachtung der KIRILlowschen Banden ${ }^{1,4}$ unumgänglich.

Da die durch Interferenzen verursachte Feinstruktur und die Kirrilowschen Banden dieselbe Erscheinung bieten, beide sich durch $\mathrm{Gl}$. (1) beschreiben lassen und unter denselben Voraussetzungen $\mathrm{zu}$ beobachten sind, muß man folgern, daß die Kirrilowschen Banden durch Interferenzen verursacht worden sind.

5 A. VAšiČEk, Optics of Thin Films, North-Holland Publ. Co., Amsterdam 1960.

\section{Zur sehr schnellen Nachwirkung des Seignettesalzes}

\section{G. KLEIN * und G. LUTHER}

Institut für Experimentalphysik II der Universität Saarbrücken

(Z. Naturforsch. 25 a, 1159-1160 [1970] ; eingegangen am 5. Juni 1970)

An Seignettesalz wurden bisher sechs verschiedene Dispersionen der Dielektrizitätskonstante $\varepsilon^{*}$ (DK) gefunden: die eigentliche ferroelektrische Relaxation ${ }^{1}$, der Abfall der DK durch den Piezoeffekt ${ }^{2}$ und vier unterscheidbare Störstellen-Relaxationen. Die Zeitkonstanten der durch Baufehler bedingten Nachwirkungen liegen bei Zimmertemperatur ungefähr bei $2 \mathrm{~min}, 7 \mathrm{~s}$,

Sonderdruckanforderungen an Dipl.-Phys. G. LUTHER, In stitut für Experimentalphysik II der Universität des Saarlandes, D-6600 Saarbrücken, Universität, Bau 4.

* Jetzt: Institut für Physikalische Chemie, II. Ordinariat, Universität Mainz.

1 H. E. Müser u. J. Pottharst, Phys. Stat. Sol. 24, 109 [1967].
$0,5 \mathrm{~ms}^{3}$ und $0,2 \mu \mathrm{s}^{4}$. Die ,schnelle“ (0,5 ms) und die „sehr schnelle Nachwirkung“ $(0,2 \mu \mathrm{s})$ wurden kürzlich von UNRUH und SAILER ${ }^{5}$ eingehender untersucht. Wir können ihre Ergebnisse bestätigen und teilen ergänzende Messungen zur sehr schnellen Nachwirkung (SSNW) am freien und geklemmten Kristall im oberen parelektrischen Bereich mit.

Im unteren parelektrischen Bereich läßt sich die bei konstanter mechanischer Spannung auftretende SSNW durch einen Debye-Formalismus ${ }^{5}$

bzw.

$$
\varepsilon^{*}=\varepsilon^{\prime}-j \varepsilon^{\prime \prime}=\varepsilon_{\infty}+\frac{\varepsilon_{\mathrm{s}}-\varepsilon_{\infty}}{1+j \omega \tau_{\varepsilon}}
$$

$$
\beta^{*}=1 / \varepsilon^{*}=\beta_{\infty}-\frac{\beta_{\infty}-\beta_{\mathrm{S}}}{1+j \omega \tau_{\beta}}
$$

2 W. P. MAson, Piezoelectric Crystals and their Application to Ultrasonics, van Nostrand, New York 1950.

3 H.-G. Unruh, Z. Angew. Physik 16, 315 [1963].

4 J. Albers, Diplomarbeit, Münster 1965.

5 H.-G. UnRuh u. E. SAILER, Z. Physik 224, 65 [1969]. 
beschreiben. Die beiden Zeitkonstanten $\tau_{\varepsilon}$ und $\tau_{\beta}$ sind mit den hoch- und niederfrequenten Grenzwerten der Dispersion $\varepsilon_{\infty}=1 / \beta_{\infty}$ und $\varepsilon_{\mathrm{s}}=1 / \beta_{\mathrm{s}}$ über die Relation

$$
\tau_{\beta}=\frac{\varepsilon_{\infty}}{\varepsilon_{\mathrm{s}}} \tau_{\varepsilon}
$$

miteinander verknüpft, wobei nur die Größe $\tau_{\beta}$ einer Arrhenius-Beziehung

$$
\tau_{\beta}=\tau_{\infty} \exp \{\Delta W / k T\}
$$

genügt. Die von uns aus Abb. 1 berechnete Aktivierungsenergie $\Delta W=(0,54 \pm 0,02) \mathrm{eV}$ liegt am Rande der Fehlergrenze des von UNRUH und SAILER ${ }^{5}$ angegebenen Wertes von $\Delta W=(0,51 \pm 0,015) \mathrm{eV}$.

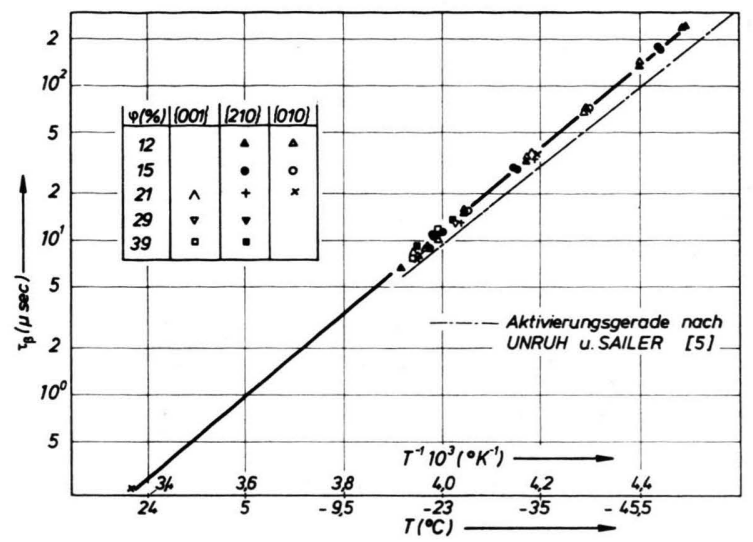

Abb. 1. Zeitkonstante $\tau_{\beta}$ der sehr schnellen Nachwirkung in Abhängigkeit von der Temperatur bei verschiedenen relativen Feuchten $\varphi$ und Wachstumsgebieten.

Extrapoliert man die am freien Kristall gefundenen Zeitkonstanten $\tau_{\beta}$ nach (4) in den oberen parelektrischen Bereich bis etwa $25{ }^{\circ} \mathrm{C}$ - im ferroelektrischen Gebiet sind die Nachwirkungen von Domänenwandbewegungen überlagert -, so ist eine Relaxationsfrequenz von ungefähr $0,5 \mathrm{MHz}$ zu erwarten. Um Störungen durch die starken piezoelektrischen Resonanzen zu vermeiden, müssen extrem kleine Proben verwendet werden. Die Abb. 2 zeigt die SSNW eines Kristallsplitters von ungefähr $0,2 \mathrm{~mm}$ Kantenlänge, dessen Vakuumkapazität $C_{0}$ zwar nicht exakt zu bestimmen ist, aber im weiteren auch nicht mehr benötigt wird. Mit wachsender Frequenz biegt die anfängliche DebyeKurve in zwei Vorresonanzen ab, bevor die eigentliche piezoelektrische Grundresonanz bei etwa $3 \mathrm{MHz}$ erreicht wird. Dennoch reicht der ungestörte Dispersionsanteil aus, um die Zeitkonstante $\tau_{\beta}$ aus den DebyeGleichungen zu ermitteln. Durch Division des Real- und Imaginärteiles von (1) folgt:

$$
\varepsilon^{\prime \prime} / \omega=\tau_{\varepsilon}\left(\varepsilon^{\prime}-\varepsilon_{\infty}\right) .
$$

Aus der Darstellung von $\varepsilon^{\prime \prime} C_{0} / \omega$ gegen $\varepsilon^{\prime} \cdot C_{0}$ erhält man nach Gl. (5) $\tau_{\varepsilon}$ und $\varepsilon_{\infty} \cdot C_{0}$, mit Hilfe von (1) und (3) ergibt sich der Wert $\tau_{\beta}=0,25 \mu \mathrm{s}$. Dieser ist in Abb. 1 eingetragen und liegt auf der zum oberen paraelektrische Bereich fortgesetzten Aktivierungsgeraden. Die SSNW existiert also auch im oberen paraelektrischen Gebiet.

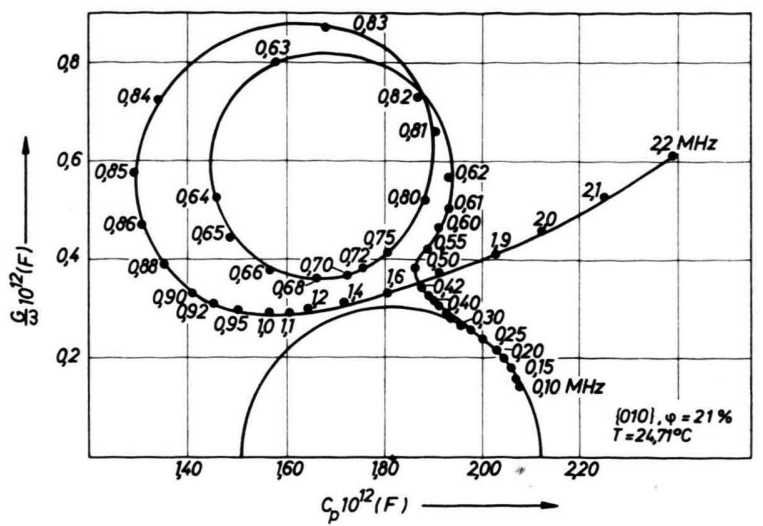

Abb. 2. Sehr schnelle Nachwirkung im oberen parelektrischen Bereich mit zwei störenden Vorresonanzen.

Das Frequenzverhalten der geklemmten DK in diesem Temperaturbereich wurde an Scherschwingern von etwa 2,2 cm Kantenlänge untersucht, deren piezoelektrische Resonanzen im interessierenden Frequenzgebiet der SSNW bereits abgeklungen sind. Die Messung erfolgte bei $24,6{ }^{\circ} \mathrm{C}$ an einem bei $12 \%$ Luftfeuchtigkeit gelagerten Kristall. Extrapoliert man aus den am freien Kristall ermittelten Werten, so wäre die SSNW bei $0,5 \mathrm{MHz}$ mit einer Dispersionsstufe (s. Abb. 9 von ${ }^{5}$ ) $\beta_{\infty}-\beta_{\mathrm{s}} \geqq 3 \cdot 10^{-4}$ zu erwarten, d. h. die DK müßte z. B. von 240 auf 225 abnehmen. Wie Abb. 3 zeigt,

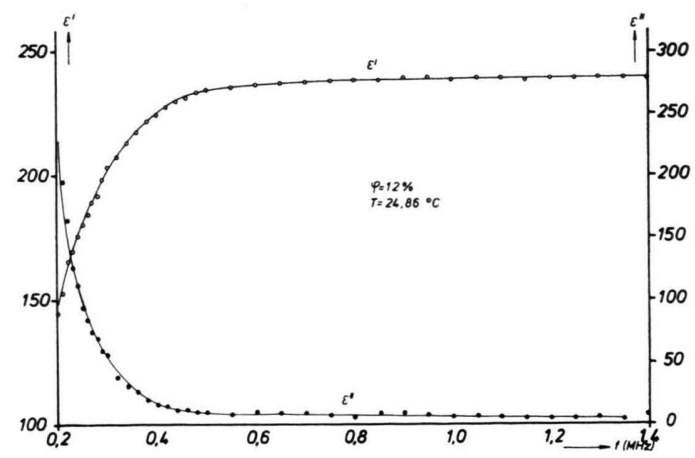

Abb. 3. Real- und Imaginärteil der DK eines im Frequenzbereich der SSNW geklemmten Kristalls.

bleibt aber der Realteil der geklemmten DK konstant und der Imaginärteil der DK deutet keine Absorption an. Der Anstieg des Realteils und der Abfall des Imaginärteils bei niedrigen Frequenzen sind von dem Ausläufer der piezoelektrischen Resonanzen verursacht.

Durch den quantitativen Nachweis der SSNW der freien DK auch im oberen paraelektrischen Bereich und das Ausbleiben der SSNW der geklemmten DK wird bestätigt, daß die Scherung als phänomenologische Ursache für die Einstellung eines eingeprägten relaxierenden Feldes ${ }^{3,5}$ der SSNW angesehen werden muß.

Unser herzlicher Dank gilt Herrn Prof. Dr. H. E. MüSER für die Anregung zu dieser Arbeit und viele wertvolle Diskussionen. 